\title{
Experiencias en el proceso de enseñanza y aprendizaje desde la discapacidad en docentes universitarios
}

\author{
Experiences in the process of teaching \\ and learning from the disability in \\ university teachers
}

DOI: http://dx.doi.org/10.17981/cultedusoc.12.2.2021.04

Recibido: 4 de mayo de 2020. Aceptado: 18 de noviembre de 2020. Publicado: 1 de julio de 2021.

\author{
Nancy Janneth Molano-Tobar \\ Universidad del Cauca. Popayán (Colombia) \\ najamoto@unicauca.edu.co
}

Luz Marina Chalapud-Narváez

Corporación Universitaria Autónoma del Cauca. Popayán (Colombia)

luz.chalapud.n@uniautonoma.edu.co

\author{
Andrés Felipe Villaquirán-Hurtado \\ Universidad del Cauca. Popayán (Colombia) \\ avillaquiran@unicauca.edu.co
}

Para citar este artículo:

Molano-Tobar, N., Chalapud-Narváez, L. y Villaquirán-Hurtado, A. (2021). Experiencias en el proceso de enseñanza y aprendizaje desde la discapacidad en docentes universitarios. Cultura, Educación y Sociedad, 12(2), 65-78. DOI: http://dx.doi.org/10.17981/ cultedusoc.12.2.2021.04

\section{Resumen}

En el artículo se analizan las experiencias educativas de docentes universitarios que orientan el proceso de actividad física a personas con discapacidad en las instituciones de educación superior. Se aborda un enfoque cualitativo, tipo estudio de caso, con diez docentes que cumplieron los criterios de inclusión. Surgen como resultado tres categorías emergentes por parte de los profesores, donde el miedo es una realidad subjetiva que limita el desempeño para las clases de Actividad Física, de la misma manera se evidenció que el conocimiento y la experiencia, se integran en el abordaje didáctico de personas con discapacidad, y desde sus narrativas consideran que la inclusión educativa es primordial para la trasformación de paradigmas propios de los actores involucrados. A manera de conclusión se plantea que la educación inclusiva es un reto tanto personal como profesional, y requiere trasformaciones del currículo, de las instituciones; todo ello influye en aspectos emocionales del ser, representando la actividad física un proceso que contribuye a fortalecer prácticas educativas que redundan en el beneficio individual y colectivo.

Palabras clave: Educación; inclusión; actividad Física; discapacidad; docentes

\section{Abstract}

The article analyzes the educational experiences of university teachers who guide the process of physical activity for people with disabilities in higher education institutions. A qualitative approach, type case study, is approached, with ten teachers who met the inclusion criteria. As a result, three emerging categories emerge on the part of the teachers, where fear is a subjective reality that limits performance for Physical Activity classes, in the same way it was evidenced that knowledge and experience are integrated into the didactic approach of people with disabilities, and from their narratives they consider that educational inclusion is essential for the transformation of paradigms of the actors involved. By way of conclusion, it is stated that inclusive education is both a personal and professional challenge, and requires transformations of the curriculum, of the institutions; All of this influences emotional aspects of being, representing physical activity a process that contributes to strengthening educational practices that result in individual and collective benefit.

Keywords: Education; inclusion; Physical activity; disability; teachers 


\section{INTRODUCCIÓN}

La discapacidad se ha convertido en un asunto relevante, permitiendo el reconocimiento de las necesidades y problemáticas entorno a esta población. Es así como en Colombia se han planteado la construcción de políticas públicas, considerando a la discapacidad un área de interés social.

Las disposiciones y normativas mundiales mencionadas por Jin, Yun \& Wegis (2013) demuestran la instauración de políticas de inclusión educativa en los distritos escolares, es así como en el ámbito Colombiano esta implícito que las facultades de Educación "deberán garantizar el desarrollo de programas de formación sobre educación inclusiva” (Ministerio de Educación Nacional-MEN, 2009); es decir, que los programas o planes cursos deberán incluir aspectos que vinculen la forma de atención, a este tipo de población, pero de la misma manera se desconoce, los procesos que se han llevado a cabo con esta población como las metodologías involucradas en la atención.

Lo anterior, genera la necesidad de realizar un análisis conceptual sobre la expresión discapacidad, desde el componente biológico al funcional, como lo indican Lugo y Seijas (2012), al evidenciar que este ha "evolucionado junto con la humanidad, desde una visión animista (castigo divino o posesión diabólica) hasta la explicación científica y el reconocimiento de los derechos", permitiendo desarrollar un proyecto de vida y participar en comunidad, logrando así estar incluidos socialmente en el ámbito familiar, escolar y laboral. Las diversas conceptualizaciones permiten advertir una serie de componentes ideológicos que transversalizan y conjugan implicaciones históricas las cuales se estructuran desde condiciones discursivas, socioculturales, políticas y económicas (Yarza, Sosa \& Pérez, 2019).

La realidad de las personas con discapacidad, es que se enfrentan diariamente a situaciones que limitan su acceso tanto estructurales como sociales, lo que incide en palabras de Misischia (2018), en su autonomía y participación en actividades cotidianas, desde sus vivencias e historias, hechos que se acentúan a la hora de participar en clases como son las relacionadas con el deporte y la actividad física, es por ello que se plantea indagar acerca de dichas vivencias a la hora de participar en diversas clases de actividad física formativa de una universidad de la ciudad de Popayán (Colombia), para propiciar a futuro estrategias de integración educativa desde la diversidad, basado en la preparación de docentes para un mundo cambiante (LePage et al., 2010).

Indudablemente las condiciones en materia de salud pública son prioritarias, razón por la cual en Colombia se ha establecido una política pública para la atención a las personas con discapacidad, tomando como base la inclusión educativa, apalancadas jurídicamente, desde la Constitución Política de 1991, la Ley 30 (1992), Ley 115 (1994), Ley 361 (1997), Ley 324 (1996), el Decreto 2082 (1996), la Ley 1618 (2013), entre otras normatividades.

Según Padilla (2014), la inclusión educativa es una forma de responder a la diversidad, aprendiendo de la diferencia y enfocándose en aprender a capitalizar las experiencias derivadas de esa diferencia, permitiendo propiciar un incentivo para fomentar el aprendizaje en la población. 
Con lo anterior, es necesario involucrar dentro del aula herramientas que propicien cambios en el contenido de las mallas curriculares, en los ambientes de trabajo y por supuesto en el materiales de aprendizaje, como preparación de los docentes (González \& Triana, 2018), situación que debe combinar acciones desde las instituciones como por parte de los mismos profesores.

Para promover una verdadera calidad educativa como lo plantea González \& Triana (2018), es necesario dentro del aula generar herramientas que propicien "cambios pertinentes en los contenidos curriculares, instalaciones locativas, materiales de aprendizaje y preparación de los docentes" (p. 203), situación que debe combinar acciones desde las instituciones como por parte de los mismos docentes, para promover una verdadera calidad educativa.

De acuerdo a las apreciaciones de Pibaque, Baque, Ayón y Ponce (2019) la educación inclusiva debe ser abordada desde una multidimensionalidad, lo que obliga a que todo docente tenga las habilidades de generar trasformaciones importantes de los contenidos curriculares orientados (Vrasmas, 2014).

Se ha logrado determinado que la actividad física genera múltiples beneficios no solo desde el componente biológico sino también, desde el ámbito psicológico como social (Barg, Armstrong, Hertz \& Laminer, 2010), es por ello que desde la clase de actividad física se pretende, "plantear modalidades de formación que promuevan un aprendizaje activo, la participación grupal y que planteen contenidos coherentes con las necesidades individuales" (Lamoneda, 2018, p. 67), hecho que permite realzar la necesidad de vincular la actividad física como una herramienta para la inclusión educativa y precisar aspectos que repercutan en la persona con discapacidad desde su esfera social, cultural y física, ante ello es que se planteo la pregunta de Investigación ¿Cuáles son las experiencias educativas de un grupo de docentes universitarios que dirigen actividad física formativa a personas con discapacidad en Popayán - Colombia?

\section{Metodología}

La investigación de la cual se deriva el artículo se enmarcó en un enfoque cualitativo, considera como referente principios teóricos que se orientan a profundizar casos específicos y no a generalizar los fenómenos percibidos de una situación (Guerrero, 2016); de la misma manera se ha basado en estudio de caso, desde los parámetros indicados por Stake (1999), donde se analiza el objeto de estudio dentro de su contexto de la vida real (experiencias educativas en la clase de Actividad Física o deporte formativo); es así como esta investigación se enfoca hacia un paradigma cualitativo interpretativo.

\section{Participantes}

En el estudio participaron 10 docentes de una Institución de Educación Superior, quienes cumplieron los parámetros de inclusión como: experiencia docente universitaria de tiempo completo, mayor de 3 años orientando clases de Actividad Física o Deporte Formativo (DF) en cualquier modalidad, quienes tuvieran matriculados estudiantes con algún tipo de discapacidad, y que de la misma manera aceptaran y firmaran el consentimiento informado como que permanecieran en todo el proceso voluntariamente. 
Dentro de los criterios de exclusión se tuvo en cuenta aquellos profesores que no aceptaran realizar el proceso investigativo o presentaran ausencias en las actividades planteadas. De la misma manera se les garantizó el manejo ético y anónimo, con la asignación de códigos de análisis regidos por números y el relato por la letra $R$, seguido por la sesión en que se realizó, lo que permitió desarrollar las observaciones.

\section{Procedimiento}

La recolección de la información se llevó a cabo en una Institución de Educación Superior (IES) entre los meses de noviembre de 2018 a febrero de 2019, mediante solicitud de participación al área de bienestar universitario como a los docentes que daban clase en los programas de Actividad Física Formativa (AFF) o Deporte Formativo (DF).

En primer lugar se tuvó una aproximación al contexto institucional de los profesores, donde se realizaron observaciones directas, entendiendo que "implica focalizar la atención de manera intencional, sobre algunos segmentos de la realidad que se estudia, tratando de capturar sus elementos constitutivos y la manera como interactúan entre sí, con el fin de reconstruir inductivamente la dinámica de la situación" (Bonilla-Castro \& Rodríguez, 2005, p. 118), donde se tuvo en cuenta el abordaje metodológico de los docentes, la interacción con los estudiantes, evidenciando las estrategias verbales y no verbales, todo ello se registro en diarios de campo, que permitieron monitorizar el proceso de observación desde una metodología descriptiva (Monistrol, 2007), enfocándose en explicar el desarrollo de las actividades del profesor y la interacción con el estudiantado.

Avanzando en el proceso se desarrollaron la entrevistas en profundidad, que en palabras de Varguillas \& Ribot (2007) se concibe como una interacción social donde se genera una comunicación de significado que da pie a relacionar las prácticas que desarrollaron os profesores con estudiantes con discapacidad durante la clase de AFF o DF, como análisis de los temas desarrollados dentro de los contenidos temáticos y las adaptaciones que realizaron para las clases.

Las entrevistas en profundidad fueron asumidas por personal capacitado, fomentando la discusión frente a las experiencias docentes y metodologías aplicadas con estudiantes con discapacidad desde la actividad física, las cuales abordaron un cuestionario semiestructurado a través de 800 horas de grabación

La sistematización de la información se centro en la identificación de unidades de análisis que permitieron delimitar en forma general el objetivo de la investigación, posteriormente se procedió a develar las categorías de análisis contrastando con la teoría, lo cual conllevó a extraer las concepciones reiteradas en el discurso de las entrevistas y así identificar las temáticas generales de las entrevistas en profundidad, derivando al proceso de triangulación que permitiera construir una explicación del fenómeno estudiado (Hernández, Fernández y Bartista, 2014), una vez teniendo las categorías emergentes se validó la pertinencia, obteniendo tres categorías: El miedo una realidad subjetiva que limita el desempeño para las clases de Actividad Física; El conocimiento y la experiencia, una mezcla para el abordaje de personas con discapacidad; La inclusión educativa primordial para la trasformación de paradigmas propios y de las personas con discapacidad. 


\section{Análisis de datos}

Se contó con el software MAXQDA (versión 12) para Windows, donde se trascribieron de manera literal las entrevistas, y los diarios de clase, para descubrir las categorías como temas esenciales del fenómeno, que fueron depuradas mediante las técnicas de triangulación, obteniendo las categorías de análisis, que dieron origen a la codificación selectiva desarrollando las ideas finales para describirlo e interpretarlo.

\section{Aspectos ético-legales}

Teniendo en cuenta la reglamentación mundial en los procesos investigativos con seres humanos, el proyecto fue evaluado y avalado por el comité de ética de la Universidad del Cauca (Colombia), mediante código de identificación 4741, de la misma manera se tuvo en cuenta lo dispuesto en la Declaración de Helsinki (Asociación Médica Mundial, WMA, 1975) y la Resolución 8430 (1993) del Ministerio de Salud y Protección Social colombiano para investigación con personas. Al grupo de apoyo se le ofrecieron charlas sobre los aspectos éticos y cómo debían asumirse los abordajes y encuentros, se presentó el proyecto a los docentes, una vez explicado y comprendido, se diligenció y aceptó mediante firma el formato de Consentimiento Informado, donde se garantiza la protección a la intimidad, a la confidencialidad como al derecho de anonimato de los datos suministrados de las entrevistas, considerando el estudio con riesgo mínimo de lesión para los participantes, de la misma manera se tuvo en cuenta lo dispuesto en la Ley 1581 (2012), acerca de la protección de los datos personales acerca de la protección de los datos personales.

\section{REsultados}

Los hallazgos en la población relacionados con la caracterización están asociados a la edad media de los docentes participantes que fue de $41.64 \pm 6.31$ años, con un tiempo de vinculación en docencia de $8.45 \pm 3.53$ años, y un promedio de $4.54 \pm 1.29$ de clases orientadas por semestre.

El análisis de los elementos derivados de las entrevistas y observaciones evidenció tres categorías finales, las cuales se presentarán a continuación:

\section{El miedo una realidad subjetiva que limita el desempeño para las clases de Actividad Física}

Dentro de los relatos y entrevistas, los docentes manifestaron que una de las limitaciones presentes en los estudiantes con discapacidad, es el hecho de enfrentarse a realizar actividades físicas desde cualquier modalidad, ya que el miedo o temor a salir lesionados o adquirir otra discapacidad permanece latente en ellos, "[...] y me dicen profe en realidad esto de hacer ejercicio me da como miedo, que tal me dañe más de lo que estoy ... ahí si joche" (R4P5). 
Lo anterior evidencia que existe un miedo de fondo con relación a su seguridad, sustentado que a pesar de los indicaciones que se les presenta, por ello el miedo al enfrentarse a programas de actividad física o deporte formativo genera una barrera más que física, la cual se convierte en una limitante emocional como psicológica "[...] las primeras clases es un trabajo arduo, donde toca realizar ejercicios de acercamiento lento pero progresivo, es más bien una trasformación de sus obstáculos, me siento combatiendo el miedo al inicio del semestre" (R12P9), al igual dichos limitantes como los temores, se encuentran configurados desde las propias experiencias de los estudiantes "un estudiante me dijo [...] uyy yo me atreví un día a meterme a una piscina, creyendo que podía, pero si no fuera por un familiar no estaría contándola, por eso me da como miedito volver a intentarlo" (R25P3).

El sentido anterior demuestra que el trabajo comienza derribando barreras y experiencias vividas por parte de los estudiantes que se inscriben a las clases de AFF, y propicia por parte del docente un cambio en las estructuras mentales de los estudiantes, lo que obliga a generar cambios en los procesos didácticos como en los acercamientos durante la clase, con la utilización de nuevas o trasformadas herramientas pedagógicas.

\section{Otra de las categorias develadas corresponde al conocimiento y la experiencia, una mezcla para el abordaje de personas con discapacidad.}

En correspondencia con esta categoría se identifica que la estructura curricular de las instituciones educativas, plantean una serie de herramientas para desarrollar con calidad la enseñanza, pero de acuerdo a los docentes, en el momento de enfrentar una clase para personas con discapacidad, se manifiesta una combinación que requiere, experiencia y creatividad, "en la universidad son pocas las herramientas que le brindan a uno para hacer actividades con personas con discapacidad, eso se gana con la experiencia y de cuenta propia, ya que la motivación propicia que uno desarrolle cosas que nunca pensó plantear para llevar a cabo sus objetivos de clase" (R2P5).

Es así como el desconocimiento de las situaciones que vive una persona con discapacidad, con relación al desenvolvimiento físico, proporciona ya un hecho que la experiencia permite superar "[..] yo ni sabía cómo se manejaba una silla de ruedas, solo fue cuando me toco ayudar a un estudiante a bajar y subir de ella para saber cómo cerrarla, abrirla hasta subir y bajar de una rampa, [...] eso lo logre con la práctica y untándome, de otra manera nada" (R25P1); en consecuencia esto indica que al vincular a las clases personas con discapacidad, se hace necesario la implementación de acciones que fomenten un vínculo como entendimiento de las realidades, partiendo de experiencias vividas hacia la implementación de procesos formativos como de actualización en pro de la personas con discapacidad, lo que determina que el docente ajuste con el tiempo de su practica docente elementos adicionales que la experiencia media a la hora de intervenir en pro de la dinámica de la clase como para el beneficio de los estudiantes.

En cuanto a los resultados identificados por parte de los profesores se pudo establecer que la inclusión educativa es primordial para la trasformación de paradigmas propios y de las personas con discapacitadas. 
Para ilustrar lo anterior se alude que, la inclusión educativa ha representado que las personas con discapacidad tengan un grado mayor de accesibilidad, pero ello también requiere que los docentes generen trasformaciones propias "[...] el trabajo con estudiantes es duro, [...] ahora dar una clase a chicos con limitaciones física, es otro mundo, me obliga a cambiar todo, mis barreras en cuanto a lo que pienso y siento hasta la metodología de cómo hacer que desarrollen una técnica específica y disfruten de la actividad física" (R14P7).

De acuerdo con lo expresado anteriormente, se induce a que las instituciones generen cambios en su infraestructura física "en los baños del centro deportivo no había duchas para personas con discapacidad, y al comenzar a llegar estudiantes con esas condiciones, la universidad debió tomar medidas, [...] pero también ha sido una lucha con los otros estudiantes que se adueñan de esos espacios, [...] todo ha conllevado a un trabajo constante de consciencia y perseverancia" (R23P8).

Como se nota en el apartado anterior, la conciencia sobre los espacios para personas con discapacidad algunas veces se ven usados, lo que genera también acciones educativas como de inclusión social al resto de estudiantes de la institución.

No obstante se ha podido revelar que las clases de AFF, promueven cambios en los estudiantes con discapacidad, fomentando cambios en la percepción como el sentir y ello se ve reflejado en las mismas clases, al notar cambios en sus auto-conceptos y autoimagen "es notorio el cambio y beneficios que las clases generan es estos chicos, pues uno al principio los ve temerosos y con muchas barreras, pero al final es otro cuento, hasta el punto de querer vincularse a los semilleros deportivos universitarios, como decir profe esto me cambio, me siento más segura y con ganas de más" (R32P2), en este sentido se aprecia como la clase de AFF promueve una cambio que trasciende lo curricular y vinculando aspectos sociales como emocionales, lo que acentúa que la actividad física repercute en las diferentes esferas de la persona con discapacidad, consolidando un pre saber que se armonizara en la discusión.

\section{DisCUSIÓN}

EL trabajo ha permitido evidenciar el sentir y vivencias de unos docentes universitarios frente a sus clases de Actividad Física o deporte formativo con personas con discapacidad, emprendiendo un entendimiento que la discapacidad es permeada por factores extrínsecos como intrínsecos, que en ciertos momentos limita la expresión corporal del que la vive (Guerrero, 2018), lo cual concuerda con lo expuesto por Zúñiga (2018), al mencionar que el cuerpo se ha convertido en una representación social de la corporalidad, lo que incide en sus procesos vitales como de salud.

La concepción anterior, facilita el entendimiento que se manifiesta con el hecho que se relaciona al temer por lo que pueda pasar al aventurarse a realizar actividades físicas, lo que indica que los aspectos emocionales como el miedo a lo nuevo o desconocido, son un factor primordial a la hora de orientar una clase (Robinson, 2016).

Es claro que en muchas veces las emociones sobrepasan las barreras físicas, pero así mismo con apoyo se logran modificarlas (Curbelo, Zarco, Almodovar, Florez \&Carmona, 2017), pues el miedo como todas las emociones son considerados como una experiencia 
vivida desde la subjetividad mediada por la cultura (Calderón, 2014), que limita en muchas ocasiones cambiar rutinas o hábitos, que es lo que los docentes del estudio manifiestan al determinar que los estudiantes con discapacidad son presa del miedo a la hora de enfrentarse a lo desconocido, lo que evidencia que el docente acceda a asumir diversas estrategias para vencer dichos obstáculos (Pollitt \& Oldfield, 2017).

Cabe considerar que la deserción académica indicada por De La Peña (2014), es aquel proceso donde el estudiante desfallece o se pierde del espacio académico, y utilizado en varias ocasiones por las personas con discapacidad, que va en contra de los que para ellos consideran un camino esencial en la inclusión educativa, ello se complementa con las palabras de Abrigo (2019), identificando que las "expresiones de la negatividad de la conciencia se alzan como llamado a la esperanza, entendida esta como dimensión trascendental que también habita en la subjetividad" (p. 6), lo cual sustenta que el miedo es una posibilidad de crecer, confirmando los beneficios que argumentan varios autores sobre la actividad física desde el componente psicosocial.

Dentro del marco de las actuales normativas mundiales como Colombianas, establecen la necesidad de vincular a los ambientes educativos a las personas con discapacidad, lo cual obliga a que los docentes estén a tono con las actuales tendencias de educación y a la vez modifiquen sus prácticas pedagógicas hacia un aprendizaje con calidad (LePage et al., 2010), lo cual se asocia a los planteamientos educativos emergentes, que buscan estrategias metodológicas integradoras con significado y participación (Williamson \& Rodríguez, 2010).

Lo anterior refleja, que se debe priorizar la capacitación del personal docente para trabajar y desarrollarse en salas de clase con un alto grado de diversidad (San Martín, Villalobos, Muñoz y Wyman, 2017), pero dicha responsabilidad no solo implica al docente, sino también plantea la necesidad que las instituciones realicen acciones hacia la actualización de su personal entorno a estos temas (Palomero, 2006) y no solo desde la voluntad o motivación de los profesores en generar ofertas para sus estudiantes con discapacidad (Bjørnsrud \& Nilsen, 2011).

Desde los planteamientos de González y Triana (2018) se evidencia que el clima en el aula, requiere de "una actitud positiva de los docentes hacia la inclusión" como en la generación de entornos favorecedores de aprendizaje, por ello como lo comentan los docentes, la vinculación de estudiantes con discapacidad, los motiva a mejorar sus acciones educativas, lo que induce a un acercamiento a las necesidades del otro, en este caso de los estudiantes con discapacidad, donde se fundamente el conocimiento e información que pueda propiciar una verdadera trasformación de los currículos (Tal, 2014).

Lo anterior, conlleva a una trasformación de los paradigmas con relación a la discapacidad, que emerge desde el mismo docente al construir metodologías desde sus propias experiencias educativas (Gilham \& Tompkins, 2016), lo que entraña cambiar el currículo tradicional que ha parcializado los conocimientos contenidos en las asignaturas, desde el conocimiento del contexto de quienes se atiende.

Una mirada integral involucra, comprender el mundo y construirlo de la mano de los actores (Ferrada, 2017); de la misma manera debe orientarse el proceso de educación inclusiva en toda la población estudiantil como lo manifiesta el profesorado, pues tanto las barreras ideológicas como estructurales, hacen que los procesos se desarrollen cohe- 
rentemente, lo cual es confirmado por Mendoza (2008), quien alude que "las actitudes ante las personas con discapacidad son actualmente negativas" (p. 272), desde el uso de terminología inadecuada mediada por estereotipos, lo cual incide y amerita educación en valores, que desde el juego y el deporte se pueden fundamentar (Martínez et al., 2014)

Se reflejó en algunas entrevistas que las limitaciones en las clases de AF o DF derivan también de las mismas personas con discapacidad, al respecto tanto Barg et al. (2010) como Palma, Raiola y Tafuri (2016), establecen que ello implica aspectos de su corporalidad, asociados al autoimagen y autoconfianza, que procede del auto concepto físico, y está asociada a aquella "representación mental, compleja y multidimensional que las personas tienen de su realidad corporal" (Fernández-Bustos, González-Martí, Contreras y Cuevas, 2016), afectando así su proyección hacia el mundo.

Hay que hacer notar entonces, que la actividad física como el deporte son una base importante para el desarrollo de las personas con discapacidad y podrían llegar a ser una herramienta de intervención en el ámbito educativo (Frutos-de-Miguel, 2018), lo que para el proceso resulto evidente y demostró que desde las experiencias educativas de los docentes universitarios, la clase de actividad física o deporte formativo, promueve cambios conceptuales y actitudinales en los estudiantes, pero a la vez suscita transformaciones y apoyo por parte de las instituciones hacia la educación inclusiva (Duncan, 2001).

\section{CONCLUSIONES}

La inclusión educativa requiere de una apuesta social, las cuales albergan a los mismos docentes, instituciones educativas como la participación del estudiantado, lo cual obliga a una reconfiguración del currículo y trasformación desde el sentir y vivencias propias.

El éxito de la inclusión educativa se asocia a la capacitación ofrecida a los docentes como a la motivación que ellos manifiesten, al involucrar otras herramientas en la practica pedagógica con el fin de propiciar ambientes inclusivos saludables, ello también mediado por la experiencia y la capacidad de interactuar con los miedos y temores del estudiantado con discapacidad.

La actividad física es una herramienta de interacción social, que involucra aportes no solo en la intervención educativa, sino que desarrolla alternativas de superación en los ámbitos sociales, emocionales y de imagen corporal.

\section{REFERENCIAS}

Abrigo, C. (2019). Negatividad de La Subjetividad y Esperanza En La Antropología Introducción Para Alfaro. Theologica Xaveriana, 69(187), 1-26. https://doi.org/10.11144/ javeriana.tx69-187.nseatj

Barg, C. J., Armstrong, B., Hetz, S. \& Latimer, A. E. (2010). Physical Disability, Stigma, and Physical Activity in Children. International Journal of Disability, Development and Education, 57(4), 371-382. https://doi.org/10.1080/1034912X.2010.524417 
Bjørnsrud, H. \& Sven N. (2011). The Development of Intentions for Adapted Teaching and Inclusive Education Seen in Light of Curriculum Potential. A Content Analysis of Norwegian National Curricula Post 1980. Curriculum Journal, 22(4), 549-566. https://doi.org/10.1080/09585176.2011.627216

Bonilla-Castro, E. y Rodríguez, P. (2005). Más allá del dilema de los métodos. Bogotá, D.C.: Uniandes.

Calderón, E. (2014). Universos Emocionales y Subjetividad. Nueva Antropología, 27(81), 11-31. Disponible en https://revistas-colaboracion.juridicas.unam.mx/index.php/nueva-antropologia/article/view/16048

Curbelo, R., Zarco P., Almodóvar, R., Flórez, M. \& Carmona, L. (2017). Barriers and Facilitators for the Practice of Physical Exercise in Patients With Spondyloarthritis : Qualitative Study of Focus Groups (EJES-3D), Reumatología Clínica, 13(2), 91-96. https://doi.org/10.1016/j.reuma.2016.03.004

Duncan, M. C. (2001). The Sociology of Ability and Disability in Physical Activity, Sociology of Sport Journal, 18(1), 1-4. https://doi.org/10.1123/ssj.18.1.1

Fernández-Bustos, J.-G., González-Martí, I., Contreras, O. y Cuevas, R. (2016). Relación entre Imagen Corporal y Autoconcepto Físico en Mujeres Adolescentes. Revista Latinoamericana de Psicología, 47(1), 25-33. https://doi.org/10.1016/S01200534(15)30003-0

Ferrada, D. (2017). Formación Docente Para La Diversidad. Revista Mexicana de Investigación Educativa, 22(74), 783-811. Disponible en https:/www.comie.org.mx/revista/ v2018/rmie/index.php/nrmie/article/view/35

Frutos-de-Miguel, J. (2018). El Autoconcepto Físico Como Herramienta de Inclusión Social en el área de Educación Física. Journal of Sport \& Health Research, 10(1), 2541. Disponible en http://www.journalshr.com/papers/Vol\%2010_N\%201/JSHR\%20 V10_1_3.pdf

Gilham, C. \& Tompkins, J. (2016). Inclusion Reconceptualized: Pre-Service Teacher Education and Disability Studies in Education. Canadian Journal of Education, 39(4), 1-25. https://doi.org/ 10.1177/0741932510362200

González, Y. y Triana D. (2018). Actitudes de Los Docentes Frente La Inclusión de Estudiantes con Necesidades Educativas Especiales. Educación y Educadores, 21(2), 200-218. https://doi.org/10.5294/edu.2018.21.2.2

Guerrero, L. (2018). Subjetividad, Intersubjetividad y Corporalidad en la teoría humeana de las pasiones indirectas. Tópicos, Revista de Filosofía, 54, 61-83. https://doi. org/10.21555/top.v0i54.881

Hernández, R., Fernández, C. y Bartista, L. (2014). Metodología de la investigación. Buenos Aires: McGraw-Hill.

Jin, J., Yun, J. \& Wegis, H. (2013). Changing Physical Education Teacher Education Curriculum to Promote Inclusion. Quest, 65(3), 372-383. https://doi.org/10.1080/00336 297.2013.791869 
De La Peña, G. (2014). El Problema de la Deserción Escolar y las Posibilidades de La Investigación-Acción Participativa. Configurações, (14), 129-149. https://doi.org/10.4000/ configuracoes.2309

Lamoneda, J. (2018). Programas de Aprendizaje-Servicio En Estudiantes de Ciclo Formativo en Animación y Actividad Física. Journal of Sport and Health Research, 10(1), 65-78. Disponible en http://www.journalshr.com/index.php/issues/2018

LePage, P., S. Courey, E. J. Fearn, V. Benson, E. Cook, L. Hartmann \& S. Nielsen. (2010). Curriculum Recommendations for Inclusive Teacher Education. International Journal of Whole Schooling, 6(2), 19-45. Available from http://files.eric.ed.gov/fulltext/EJ912015.pdf

Lugo, L. y Seijas, V. (2012). La Discapacidad En Colombia: Una Mirada Global. Revista Colombiana de Medicina Física y Rehabilitación, 22(2), 164-179. http://www.revistacmfr.org/index.php/rcmfr/article/view/64

Martínez, R., Cepero, M., Collado, D., Padial, R., Pérez, A. y Palomares, J. (2014). Adquisición de Valores y Actitudes mediante el Juego y el Deporte en Educación Física, en Educación Secundaria. Journal of Sport and Health Research, 6(3), 207-216. Recuperado de http://www.journalshr.com/papers/Vol\%206_N\%203/V06_3_3.pdf

MAXQDA. (versión 12). [Software para análisis de datos cualitativos]. Freiburg: VERBI $\mathrm{GmbH}$. Disponible en https://es.maxqda.com/descargar

Mendoza, N. (2008). La Formación del Profesorado en Educación Física con relación a Las Personas con Discapacidad. Psychosocial Intervention, 17(3), 269-279. https:// doi.org/10.4321/S1132-05592008000300004

Misischia, B. S. (2018). La Relación Universidad - Discapacidad. Educación, Lenguaje y Sociedad, 15(15), 1-18. Disponible en https://cerac.unlpam.edu.ar/index.php/els/article/view/3432

Monistrol, O. (2007). El trabajo de campo en investigación cualitativa (I). Nure investigación, (28), 1-4.Disponible en https://www.nureinvestigacion.es/OJS/index.php/nure/ article/view/339

Padilla, A. (2014). Inclusión Educativa de Personas Con Discapacidad. Revista Colombiana de Psiquiatría, 40(4), 670-699. https://doi.org/10.1016/s0034-7450(14)60157-8

Palma, D., Raiola, G. \& Tafuri, D. (2016). Disability and Sport Management: A Systematic Review of the Literature. Journal of Physical Education and Sport, 16(125), 785-793. https://doi.org/10.7752/jpes.2016.03125

Palomero, J. (2006). Formación Inicial de los profesionales de la Educación en Pedagogía Intercultural: Una Asignatura Pendiente. Revista Interuniversitaria de Formación del Profesorado, 20(1), 207-230. Disponible en https://recyt.fecyt.es/index.php/RIFOP/issue/archive

Pibaque, M., Baque, L., Ayón, L. y Ponce S. (2019.) La Dinámica Educativa Intercultural y La Inclusión Educativa. Revista Lasallista de Investigación, 15(2), 153-168. https:// doi.org/10.22507/rli.v15n2a12 
Pollitt, K. \& Oldfield, J. (2017). Overcoming the Odds: Exploring Barriers and Motivations for Male Trainee Primary Teachers. Teaching and Teacher Education, 62, 30-36. https://doi.org/10.1016/j.tate.2016.11.003

República de Colombia. Congreso de la República. (27 de febrero de 2013). Por medio de la cual se establecen las disposiciones para garantizar el pleno ejercicio de los derechos de las personas con discapacidad. [Ley 1618/2013]. Diario Oficial 48.717. Encontrado en https://discapacidadcolombia.com/phocadownloadpap/LEGISLACION/LEY\%20 ESTATUTARIA\%201618\%20DE\%202013.pdf

República de Colombia. Congreso de la República. (27 de junio de 2012). Por la cual se dictan disposiciones generales para la protección de datos personales. [Ley 1581]. Diario Oficial 48.587. Recuperado de https://www.funcionpublica.gov.co/eva/gestornormativo/ norma_pdf.php?i=49981

República de Colombia. MEN. (9 de febrero de 2009). Por medio del cual se reglamenta la organización del servicio de apoyo pedagógico para la atención de los estudiantes con discapacidad y concapacidades o con talentos excepcionales en el marco de la educación inclusiva. [Decreto 366]. Diario Oficial 47.258. Disponible en https://www.mineducacion.gov.co/1621/article-182816.html

República de Colombia. MinSalud. (4 de octubre de 1993). Por la cual se establecen las normas científicas, técnicas y administrativas para la investigación en salud. [Resolución 8430]. Recuperado de https://www.hospitalsanpedro.org/images/Comite_Investigacion/Resolucion_8430_de_1993.pdf

República de Colombia. Congreso de la República. (7 de febrero de 1997). Por el cual se establecen mecanismos de integración social de las personas con limitación y se dictan otras disposiciones. [Ley 361/1997]. Diario Oficial 42.978. Recuperado de https://www. mintic.gov.co/portal/604/articles-3673_documento.pdf

República de Colombia. Congreso de la República. (11 de octubre de 1996). Por la cual se crean algunas normas a favor de la población sorda. [Ley 324/1996]. Diario Oficial 42.899. Recuperado de https://www.funcionpublica.gov.co/eva/gestornormativo/norma_pdf.php?i=349

República de Colombia. Congreso de la República. (8 de febrero de 1994). Por la cual se expide la ley general de Educación. [Ley 115/1994]. Diario Oficial 41.214. Recuperado de https://www.mineducacion.gov.co/1621/articles-85906_archivo_pdf.pdf

República de Colombia. Congreso de la Republica. (28 de diciembre de 1992). Por el cual se organiza el servicio público de la Educación Superior. [Ley 30/1992]. Diario Oficial 40.700. Recuperado de https://www.mineducacion.gov.co/1621/articles-86437_Archivo_pdf.pdf

República de Colombia. Congreso de la República. (1991). Constitución Política. [Online]. Recuperado de https://www.corteconstitucional.gov.co/inicio/Constitucion\%20politica\%20de\%20Colombia\%20-\%202015.pdf 
República de Colombia. Presidencia de la República. (20 de noviembre de 1996). Por el cual se reglamenta la atención educativa para personas con limitaciones o con capacidades o talentos excepcionales. [Decreto 2082/1996]. Diario Oficial No. 42.922. Recuperado de https://www.mineducacion.gov.co/1759/articles-103323_archivo_pdf.pdf

Robinson, G. (2016). Culturally Responsive Beliefs and Practices of General and Special Education Teachers Within a Response to Intervention Framework. Multiple Voices for Ethnically Diverse Exceptional Learners, 16(2), 22-36. Available: https:// eric.ed.gov/?id=EJ1121390

San Martín, C., Villalobos, C., Muñoz, C. y Wyman, I. (2017). Formación Inicial Docente Para La Educación Inclusiva. Análisis de Tres Programas Chilenos de Pedagogía en Educación Básica que incorpora la Perspectiva de la Educación Inclusiva. Calidad en la Educación, 46, 20-52. http://dx.doi.org/10.31619/caledu.n46.2

Stake, R. E. (1999). Investigación Con Estudio de Casos. Madrid: Morata.

Tal, C. (2014). Introduction of an Emergent Curriculum and an Inclusive Pedagogy in a Traditional Setting in Israel: A Case Study. International Journal of Early Years Education, 22(2), 141-155. https://doi.org/10.1080/09669760.2014.898578

Varguillas, C. y Ribot, S. (2007). Implicaciones conceptuales y metodológicas en la aplicación de la entrevista en profundidad. Laurus, 13(23), 249-262.

Vrasmas, T. (2014). Curriculum for Children with Disabilities in Inclusive Education. A Literature Review. Procedia - Social and Behavioral Sciences, 127, 336-341. https:// doi.org/10.1016/j.sbspro.2014.03.267

WMA. (1975). Declaración de Helsinki de la AMM - Principios éticos para las investigaciones médicas en seres humanos. [Online]. Disponible en https://www.wma.net/es/ policies-post/declaracion-de-helsinki-de-la-amm-principios-eticos-para-las-investigaciones-medicas-en-seres-humanos/

Williamson, C. \& Rodríguez, C. (2010). Equidad, Inequidad y Educación Superior: Aprendizajes de un Preuniversitario para Jóvenes de la educación de Adultos. Estudios Pedagógicos, 36(2), 251-267. http://dx.doi.org/10.4067/S0718-07052010000200015

Yarza, A., Sosa, L. \& Pérez, B. (2019). Estudios críticos en discapacidad: una polifonía desde América Latina. Buenos Aires: CLACSO. https://doi.org/10.2307/j.ctv1gm00ws

Zúñiga, Y. (2018). Cuerpo, Género y Derecho. Apuntes Para Una Teoría Crítica de Las Relaciones Entre Cuerpo, Poder y Subjetividad.” Revista Ius et Praxis, 24(3), 209254. https://doi.org/10.4067/s0718-00122018000300209

Nancy Janneth Molano Tobar. Docente del Departamento de Educación Física, Recreación y Deporte en la Facultad de Ciencias Naturales, Exactas y de la Educación de la Universidad del Cauca (Colombia). https://orcid.org/0000-0003-1953-4101

Luz Marina Chalapud Narváez. Docente del Programa de Entrenamiento Deportivo, en la Facultad de Educación de la Corporación Universitaria Autónoma del Cauca (Colombia). https://orcid.org/0000-0003-4047-7105 
Andrés Felipe Villaquirán Hurtado. Jefe del Departamento de Fisioterapia, investigador Junior categorizado por MinCiencias, coordinador de la línea de investigación en Actividad Física y Deporte e integrante del Grupo de investigación Movimiento Corporal Humano y Calidad de Vida del programa de Fisioterapia, docente en el área de la fisioterapia deportiva en programas de pregrado y posgrado. Universidad del Cauca (Colombia). https://orcid.org/0000-0002-6156-6425 